\title{
Modeling Urban Growth Patterns with Correlated Percolation
}

\author{
Hernán A. Makse ${ }^{1}$, José S. Andrade Jr. ${ }^{1,2}$, Michael Batty ${ }^{3}$, \\ Shlomo Havlin ${ }^{1,4}$ and H. Eugene Stanley ${ }^{1}$ \\ ${ }^{1}$ Center for Polymer Studies and Dept. of Physics, Boston University, Boston, MA 02215 USA \\ ${ }^{2}$ Departamento de Física, Universidade Federal do Ceará, 60451-970 Fortaleza, Ceará, Brazil \\ ${ }^{3}$ Centre for Advanced Spatial Analysis, University College London, \\ 1-19 Torrington Place, London WC1E 6BT, UK \\ ${ }^{4}$ Department of Physics, Bar-Ilan University, Ramat-Gan, Israel
}

(Phys. Rev. E (1 December 1998))

\begin{abstract}
We propose and test a model that describes the morphology of cities, the scaling of the urban perimeter of individual cities, and the area distribution of systems of cities. The model is also consistent with observable urban growth dynamics, our results agreeing both qualitatively and quantitatively with urban data. The resulting growth morphology can be understood from interactions among the constituent units forming an urban region, and can be modeled using a correlated percolation model in the presence of a gradient.
\end{abstract}

\section{INTRODUCTION}

Traditional approaches to urban science as exemplified in the work of Christaller [1], Zipf [2], Stewart and Warntz [3], Beckmann 四, and Krugman [5] are based on the assumption that cities grow homogeneously in a manner that suggests that their morphology can be described using conventional Euclidean geometry. However, recent studies have proposed [6] that the complex spatial phenomena associated with actual urban systems is rather better described using fractal geometry consistent with growth dynamics in disordered media 69 .

Predicting urban growth dynamics also presents a challenge to theoretical frameworks for cluster dynamics in that different mechanisms clearly drive urban growth from those which have been embodied in existing physical models. In this paper, we develop a mathematical model that relates the physical form of a city and the system within which it exists, to the locational decisions of its population, thus illustrating how paradigms from physical and chemical science can help explain a uniquely different set of natural phenomena - the physical arrangement, configuration, and size distribution of towns and cities. Specifically, we argue that the basic ideas of percolation theory when modified to include the fact that the elements forming clusters are not statistically independent of one another but are correlated, can give rise to morphologies that bear both qualitative and quantitative resemblance to the form of individual cities and systems of cities. Some of these results are briefly described in Ref. [10].

We consider the application of statistical physics to urban growth phenomena to be extremely promising, yielding a variety of valuable information concerning the way cities grow and change, and more importantly, the way they might be planned and managed. Such information includes (but is not limited to) the following:

(i) the size distributions of towns, in terms of their populations and areas;

(ii) the factal dimensions associated with individual cities and entire systems of cities;

(iii) interactions or correlations between cities which provide insights into their interdependence;

(iv) the relevance and effectiveness of local planning policies, particularly those which aim to manage and contain growth.

The size distribution of cities has been a fundamental question in the theory of urban location since its inception in the late 19th century. In the introduction to his pioneering book published over 60 years ago, Christaller [1] posed a key question: "Are there laws which determine the number, size, and distribution of towns?" This question has not been properly answered since the publication of Christaller's book, notwithstanding the fact that Christaller's theory of central places [i] and its elaboration through theories such the rank-size rule for cities [2. 1. embody one of the cornerstones of human geography.

Our approach produces scaling laws that quantify such distributions. These laws arise naturally from our model, and they are consistent with the observed morphologies of individual cities and systems of cities which can be characterized by a number of fractal dimensions and percolation exponents. In turn, these dimensions are consistent with the density of location around the core of any city, and thus the theory we propose succeeds in tying together both intra- and inter-urban location theories which have developed in parallel over the last 50 years. Furthermore, the striking fact that cities develop a power law distribution without the tuning of any external parameter might be associated with the ability of systems of cities to "self-organize" [0].

\section{THE DLA MODEL}

Cities grow in a way that might be expected to resemble the growth of two-dimensional aggregates of particles, and this has led recent attempts [6, 11, 12] to model urban 
growth using ideas from the statistical physics of clusters. In particular, the model of diffusion limited aggregation (DLA) 13, 14 has been applied to describe urban growth [6], and results in tree-like dendritic structures which have a core or "central business district" (CBD). The DLA model is a physical model used to describe aggregation phenomena and is related to problems from the field of oil recovery in which "viscous fingering" occurs when a low viscosity fluid is pushed under pressure into a fluid with a larger viscosity (as occurs when an oil field is flooded with water in an attempt to "push out the oil").

The DLA model predicts that there exists only one large fractal cluster that is almost perfectly screened from incoming "development units" (people, capital, resources, etc), so that almost all the cluster growth occurs in the extreme peripheral tips. However, quantitative data do not support all the properties of the DLA model. For instance, the DLA model predicts that the urban population density $\rho(r)$ decreases from the city center as a power law,

$$
\rho(r) \sim r^{D-2}
$$

where $r$ is the radial distance from the core, and $D \simeq 1.7$ is the fractal dimension of DLA. However, urban data have been more commonly fit to an exponential decay [15]. In the DLA model only one large central place or cluster is generated, while a real urban area is formed by a system of central places that are spatially distributed in a hierarchy of cities. Still another concern regarding the morphology of the DLA model is that DLA is a simplyconnected cluster. Cities grow in a more compact way, with a well-defined urban boundary or external perimeter not accounted for by the dendritic fractal growth of DLA.

Here we show that an alternative model, in which development units are correlated rather than being added to the cluster at random, is better able to reproduce the observed morphology of cities and the area distribution of sub-clusters ("towns") in an urban system, and can also describe urban growth dynamics. Our "physical" model 10], which corresponds to the correlated percolation model [16 20] in the presence of a density gradient [21 23], is motivated by the fact that in urban areas development attracts further development. The model offers the possibility of predicting the global properties (such as scaling behavior) of urban morphologies.

\section{CORRELATED PERCOLATION MODEL}

In the model we now develop, we take into account two points:

(i) First, data on population density $\rho(r)$ of actual urban systems are known to conform to the relation [15]

$$
\rho(r)=\rho_{0} e^{-\lambda r},
$$

where $r$ is the radial distance from the central business district (CBD) situated at the core, and $\lambda$ is the density gradient. The density gradient quantifies the extent of the urban spread around the central core. The probability that a unit occupies a given spot decreases gradually as the distance from a central, compact core increases.

(ii) Second, in actual urban systems, the development units are not positioned at random. Rather, there exist correlations arising from the fact that when a development unit is located in a given place, the probability of adjacent development units increases naturally; each site is not independently occupied by a development unit, but is occupied with a probability that depends on the occupancy of the neighborhood. In urban settings, development units do not attach themselves randomly to an existing cluster. Their placement is strongly influenced by the presence of other units. When a unit occupies a certain location, the probability of additional development is highest in its vicinity, and this probability decreases at a certain rate as the distance from the unit increases. Thus, the rules of placement are affected by long-range "interactions" that influence how clusters form and grow. What happens at a given site depends on the state of many other sites. These correlations reflect the tendency of people to locate next to one another, as articulated in traditional urban science as economies of urban agglomeration.

In order to quantify these ideas, we consider the correlated percolation model 16 20 in the presence of a concentration gradient 21 23. We start by describing the uncorrelated site percolation problem, which corresponds to the limit where correlations are so small as to be negligible 17 9]. We first define a random number $u(r)$, called the occupancy variable, at every site $r=(i, j)$ in a square lattice of $L^{2}$ sites. The numbers $u(r)$ are uncorrelated numbers with a uniform probability distribution between $[0,1]$. A site in the lattice is occupied if the occupancy variable $u(r)$ is smaller than the occupation probability $p$, which is a quantity fixed for every site in the lattice. A cluster is a set of sites connected via nearest neighbor sites. When $p$ is small only isolated clusters exist. At a critical value of the concentration called $p_{c}$ an "incipient infinite cluster" forms which, for a finite system, connects two sides of the system.

Our basic model is a percolation model modified to introduce correlations among the units and the fact that the concentration $p$ is not constant for all the points in the lattice but presents the gradient shown in Eq. (2). In our model we consider "development units" representing buildings, people, resources which are added to the cluster in similar fashion as in percolation. Since these units are added in a correlated fashion, we next consider a modification of the percolation problem to incorporate correlations among the occupancy variables $u(r)$.

To introduce correlations among the variables we use a method proposed in 20] which is a modification of the Fourier filtering method (Ffm) 24 26, 18 suitable for large systems. Consider a stationary sequence of $L^{2}$ 
uncorrelated random numbers $\{u(r)\}, r=(i, j), i, j=$ $1, . ., L$. The correlation function is $\left\langle u(r) u\left(r^{\prime}\right)\right\rangle \sim \delta_{r, r^{\prime}}$, with $\delta_{r, r^{\prime}}$ the Kronecker delta, and the brackets denote an average with respect to a Gaussian distribution. We use the sequence $\{u(r)\}$, to generate a new sequence, $\{\eta(r)\}$, with a long-range power-law correlation function $C(\ell)$ of the form 20

$$
C(\ell) \equiv\left\langle u(r) u\left(r^{\prime}\right)\right\rangle=\left(1+\ell^{2}\right)^{-\alpha / 2}
$$

where, $\ell=\left|r-r^{\prime}\right|, \alpha$ is the correlation exponent, and the long-range correlations are relevant for $0<\alpha<d=2$, where $d$ is the dimension of the substrate $-\alpha \geq 2$ corresponds to the uncorrelated problem, and $\alpha \rightarrow 0$ to the strongly correlated problem.

The spectral density $S(q)$, defined as the Fourier transform of $C(\ell)$, has the form

$$
S(q)=\frac{2 \pi}{\Gamma\left(\beta_{2}+1\right)}\left(\frac{q}{2}\right)^{\beta_{2}} K_{\beta_{2}}(q),
$$

where $q=|\vec{q}|, q_{i}=2 \pi m_{i} / L,-L / 2 \leq m_{i} \leq L / 2, i=1,2$, and $\beta_{2}=(\alpha-2) / 2$. $\{\eta(q)\}$ are the Fourier transform coefficients of $\{\eta(r)\}$, and satisfy

$$
\eta(q)=(S(q))^{1 / 2} u(q)
$$

where $\{u(q)\}$ are the Fourier transform coefficients of $\{u(r)\}$.

The actual numerical algorithm for Ffm consists of the following steps: (i) Generate a two-dimensional sequence $\{u(r)\}$ of uncorrelated random numbers with a Gaussian distribution, and calculate the Fourier transform coefficients $\{u(q)\}$. (ii) Obtain $\{\eta(q)\}$ using (14) and (5). (iii) Calculate the inverse Fourier transform of $\{\eta(q)\}$ to obtain $\{\eta(r)\}$, the sequence in real space with the desired power-law correlation function which asymptotically behaves as

$$
C(\ell) \sim \ell^{-\alpha}
$$

The assumption of power-law interactions is motivated by the fact that the "decision" for a development unit to be placed in a given location decays gradually with the distance from an occupied neighborhood.

Finally we consider that the development units are positioned with a probability which behaves in the same fashion as known for cities Eq. (2). Therefore we relax the assumption that the concentration $p$ is constant for all the points in the lattice, and we consider that the development units are positioned with an occupancy probability

$$
p(r) \equiv \rho(r) / \rho_{0}
$$

that behaves in the same fashion as is known in observations of real cities. This last modification corresponds to the percolation problem in the presence of a concentration gradient proposed in 21 23.

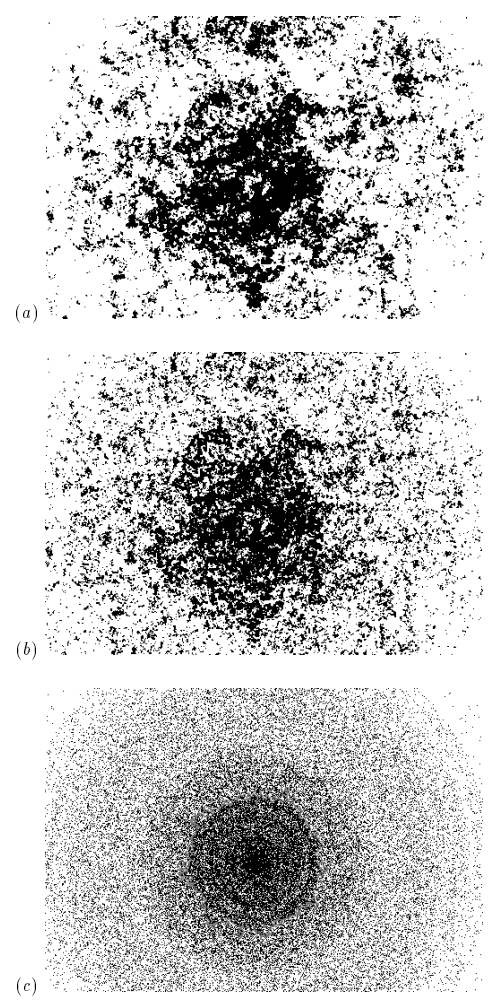

FIG. 1. Simulations of urban systems for different degrees of correlation. Here, the urban areas are red, and the external perimeter or urban boundary of the largest cluster connected to the $\mathrm{CBD}$ is light green. In all the figures, we fix the value of the density gradient to be $\lambda=0.009$. (a) and (b) Two different examples of interactive systems of cities for correlation exponents $\alpha=0.6$ and $\alpha=1.4$, respectively. The development units are positioned with a probability that decays exponentially with the distance from the core. The units are located not randomly as in percolation, but rather in a correlated fashion depending of the neighboring occupied areas. The correlations are parametrized by the exponent $\alpha$. The strongly correlated case corresponds to small $\alpha(\alpha \rightarrow 0)$. When $\alpha>d$, where $d$ is the spatial dimension of the substrate lattice $(d=2$ in our case), we recover the uncorrelated case. Notice the tendency to more compact clusters as we increase the degree of correlations $(\alpha \rightarrow 0)$. (c) As a zeroth order approximation, one might imagine the morphology predicted in the extreme limit whereby development units are positioned at random, rather than in the correlated way of Figs. $1 a$ and $1 b$. The results for this crude approximation of a non-interactive (uncorrelated) system of cities clearly display a drastically different morphology than found from data on real cities (such as shown in Fig. 2a). The non-interactive limit looks unrealistic in comparison with real cities, for the lack of interactions creates a urban area characterized by many small towns spread loosely around the core. 
In order to apply the above procedure to the percolation problem, we study the probability distribution $P(\eta)$ of the correlated sequence $\eta(r)$. We find that when the uncorrelated variables $u(r)$ are taken from a Gaussian distribution, $\eta(r)$ also has a Gaussian distribution. We next discretize the variables generating a sequence $\mu(i, j)$, according to $\mu(i, j)=\Theta(\theta-\eta(r))$ where $\theta$ is chosen to satisfy $p(r)=\int_{-\infty}^{\theta} P(\eta) d \eta$, with $p(r)$ the occupancy probability and $\Theta$ is the Heaviside step function.

Notice that we have defined two different properties of the system. First we introduced long range correlations among the variables by modifying the occupancy variables $\eta(r)$. These correlations are isotropic, i.e., all the points in space are connected by interactions quantified by a power law. The fact that we consider a slowlydecaying power-law scale-free function is due to the fact that any other correlation function will display a cut-off after which correlations are negligible. Since we are looking at properties of actual cities at large length scales, a coarse grain will transform a finite range correlated system into a uncorrelated system, i.e., a system with a finite cut-off in the correlations becomes uncorrelated at large scales. This situation occurs when we consider power law correlations of the form (6) since it is a scale-free function. Thus correlations are expected to be relevant at all length scales. One must distinguish the type of correlation introduced by (6) from the correlations arising at the critical concentration $p_{c}$. In this case, the connectedness length of the system is said to be infinite since two occupied sites separated by an arbitrary distance may be connected by the infinite cluster, and thus correlated in space. However, the correlations introduced by (6) goes beyond this type of connection between sites. Due to correlation of type (6), even sites which belong to different clusters are correlated.

Second, we consider that the probability of occupancy of the sites decays exponentially with the center point always occupied. This property of the system is independent of the type of correlation chosen. The correlation exponent $\alpha$ and the density gradient $\lambda$ are the only parameters of the model to be determined by empirical observations.

\section{STATICS}

We first discuss the influence of the correlations on the morphology of a system of cities generated in the present model. Therefore we fix the value of the concentration gradient $\lambda$ in Eq. (7) and we show in Fig. 1 our simulations of correlated urban systems for different degrees of correlation. We see that the larger the degree of correlations the more compact the clusters are. The correlations have the effect of agglomerating the units around an urban area. In the simulated systems the largest city is situated in the core (which acts as the "attractive" center of the city), and this is surrounded by small clusters or "towns." The correlated clusters are fairly compact near their respective centers and become less compact near their boundaries, in qualitative agreement with empirical data on actual large cities such as Berlin, Paris and London. (see i.e. Refs. 6, 27]). The strongly correlated case of Fig. 1 a $\alpha \rightarrow 0$ results in a system of cities looking more realistic than the uncorrelated case (Fig. 11c). The uncorrelated case results in a systems of very small cities spread around a central city, while the cities in the correlated case look more compact and more realistic.
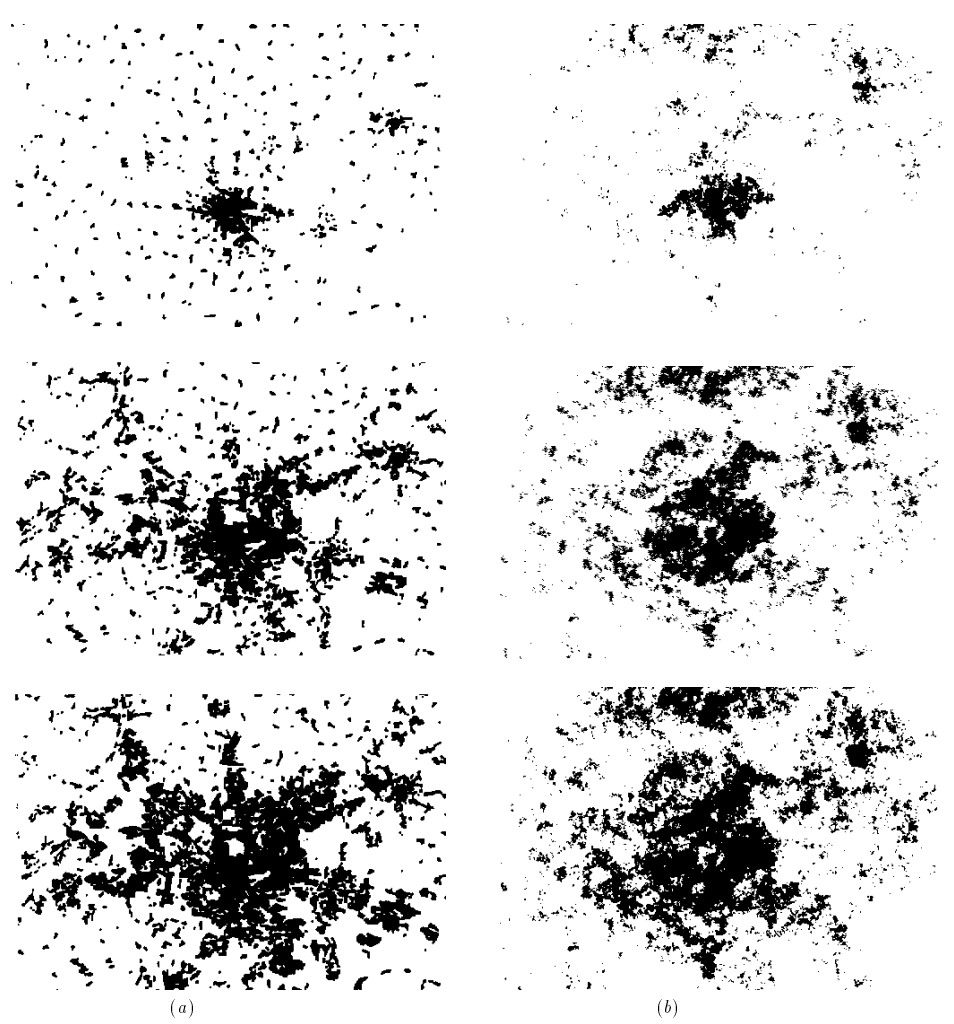

FIG. 2. Qualitative comparison between the actual urban data and the proposed model. (a) Three steps of the growth with time of Berlin and surrounding towns. Data are shown for the years 1875, 1920, and 1945 (from top to bottom). (b) Dynamical urban simulations of the proposed model. We fix the value of the correlation exponent to be $\alpha=0.05$ (strongly correlated case), and choose the occupancy probability $p(r)$ to correspond to the density profiles shown in Fig. 6. We use the same seed for the random number generator in all figures.

For distances smaller that $r_{f}$, there is a high concentration of sites since $p(r)>p_{c}$, and the cluster thus generated plays the role of the infinite cluster. For distance larger than $r_{f}$, we have $p(r)<p_{c}$, so that only isolated clusters exist, which form the system of small cities surrounding the large city situated in the core.

The urban boundary of the largest city is defined to be the external perimeter of the cluster connected to the CBD. Since $p(r)$ decreases as one moves away from the core, the probability that the largest cluster remains connected decreases with $r$. The mean distance of the 
perimeter from the center $r_{f}$ is determined by the value of $r$ for which $p(r)$ equals the percolation threshold-i.e. $p\left(r_{f}\right)=p_{c}$, so 21 23.

$$
r_{f}=\lambda^{-1} \ln \left(1 / p_{c}\right) .
$$

The geometrical properties of the external perimeter of the largest city correspond to the properties of the external perimeter of the infinite cluster of the percolation problem in the absence of a gradient [21]. The critical properties of the clusters can be analyzed in terms of the percolation properties. Percolation clusters formed below $p_{c}$ are characterized by a finite connectedness length which is the typical distance at which two sites are expected to be connected via nearest neighbor sites (do not confuse with the correlations introduced via Eq. (5)). This connectedness length diverges when the infinite cluster forms at $p_{c}$, i.e. $\xi \sim\left|p-p_{c}\right|^{-\nu}$, where $\nu$ is the connectedness length exponent. In the case of gradient percolation the clusters formed below $p_{c}$ for $r>r_{f}$ are characterized by this length, which is now a function of the distance $r$

$$
\xi(r) \sim\left|p(r)-p_{c}\right|^{-\nu} .
$$

Moreover, due to the existence of long range correlation among the variables the exponent $\nu$ is not universal, but changes continuously with the degree of correlation given by $\alpha$ [18]. We will see that several critical properties of the percolation clusters change with the correlations.

The width $\sigma_{f}$ of the external perimeter of the largest city is defined as

$$
\sigma_{f} \equiv\left\langle\left(r-r_{f}\right)^{2}\right\rangle^{1 / 2}
$$

where $r_{f} \equiv\langle r\rangle$, and $r$ belongs to the external perimeter of the central cluster. The width of the external perimeter is a function of the concentration gradient $\lambda$ and it is known to scale as [21]

$$
\sigma_{f} \sim \lambda^{-\nu /(1+\nu)}
$$

The value of $\nu$ corresponding to the uncorrelated percolation problem is $\nu=4 / 3$. However the presence of long range correlations of the type (6) drastically affects the value of the connectedness exponent, which is now a function of $\alpha, \nu(\alpha)$ as observed in previous studies of long range correlated percolation [17,18]. We have simulated the correlated percolation problem with a gradient and using Eq. (11) we find a drastic increase of $\nu(\alpha)$ with the increase of the long-range correlations $(\alpha \rightarrow 0)$ (Figs. Ba, $3 \mathrm{~b})$. In particular $\nu(\alpha)$ seems to increase very drastically for a system of strong correlations $\alpha \rightarrow 0$. In fact for such a system, we expect a mean field situation where all sites in the lattice are connected to the rest of the sites. In this case the percolation threshold for the site percolation problem should be $p_{c}=0.5$ and the connectedness length should be zero below $p_{c}$ and infinite above $p_{c}$.

The scaling of the length of the urban boundary of the largest city within a region of size $\ell$,

$$
L(\ell) \sim \ell^{D_{e}},
$$

defines the fractal dimension $D_{e}$, which we calculate to have values $D_{e} \simeq 1.33$ for the uncorrelated case, and $D_{e} \simeq 1.4$ for strong correlations $(\alpha \rightarrow 0)$ (Fig. $3 \mathrm{c}$ ). The small variation of the fractal dimension of the external perimeter does not rule out the fact that it may be independent of the correlations. These values are consistent with actual urban data, for which values of $D_{e}$ between 1.2 and 1.4 are measured [6].

Near the frontier and on length scales smaller than the width of the frontier $\sigma_{f}$, the largest cluster has fractal dimension $d_{f} \simeq 1.89$, as defined by the "mass-radius" relation

$$
M(r) \sim r^{d_{f}}
$$

where $M(r)$ is the mass of the cluster inside a region of radius $r$. The value $d_{f} \simeq 1.89$ corresponds to the fractal dimension of the uncorrelated percolation clusters and we find that it is valid independent of the correlations. However, as $\alpha \rightarrow 0$ we expect a compact cluster with dimension $d_{f}=2$. The fact that we are unable to see this limit might be due to numerical limitations near the mean field point $\alpha=0$.

The number of sites of the frontier $N_{f}$ also scales with the concentration gradient 21]

$$
N_{f} \sim \lambda^{-\nu\left(d_{f}-1\right) /(1+\nu)} .
$$

This relation provides another way of calculating the fractal dimension $d_{f}$ and the exponent $\nu$, which we used to verify our calculations.

It is important to stress that under the present percolation picture cities are fractal structures only near the external perimeter of the largest city, and on length scales smaller than the width of the frontier defined by Eq. (10). The width is a function of the concentration gradient $\lambda$, Eq. (11) so that the larger the spread of the city the larger the region where the city is fractal. However, at distances close to the center of the largest city, the cluster is clearly non fractal since $p(r)>p_{c}$ and the cluster becomes compact. On the other side for larger distances $p(r)<p_{c}$, only small isolated clusters exist with a definite connectedness length associated with them (9), so that they are not fractal either.

\section{AREA DISTRIBUTION OF URBAN SETTLEMENTS}

So far, we have argued how correlations between occupancy probabilities can account for the irregular morphology of towns in a urban system. As can be seen in Fig. Ea, the towns surrounding a large city like Berlin are characterized by a wide range of sizes. We are interested in the laws that quantify the town size distribution 
$N(A)$, where $A$ is the area occupied by a given town or "mass" of the agglomeration.
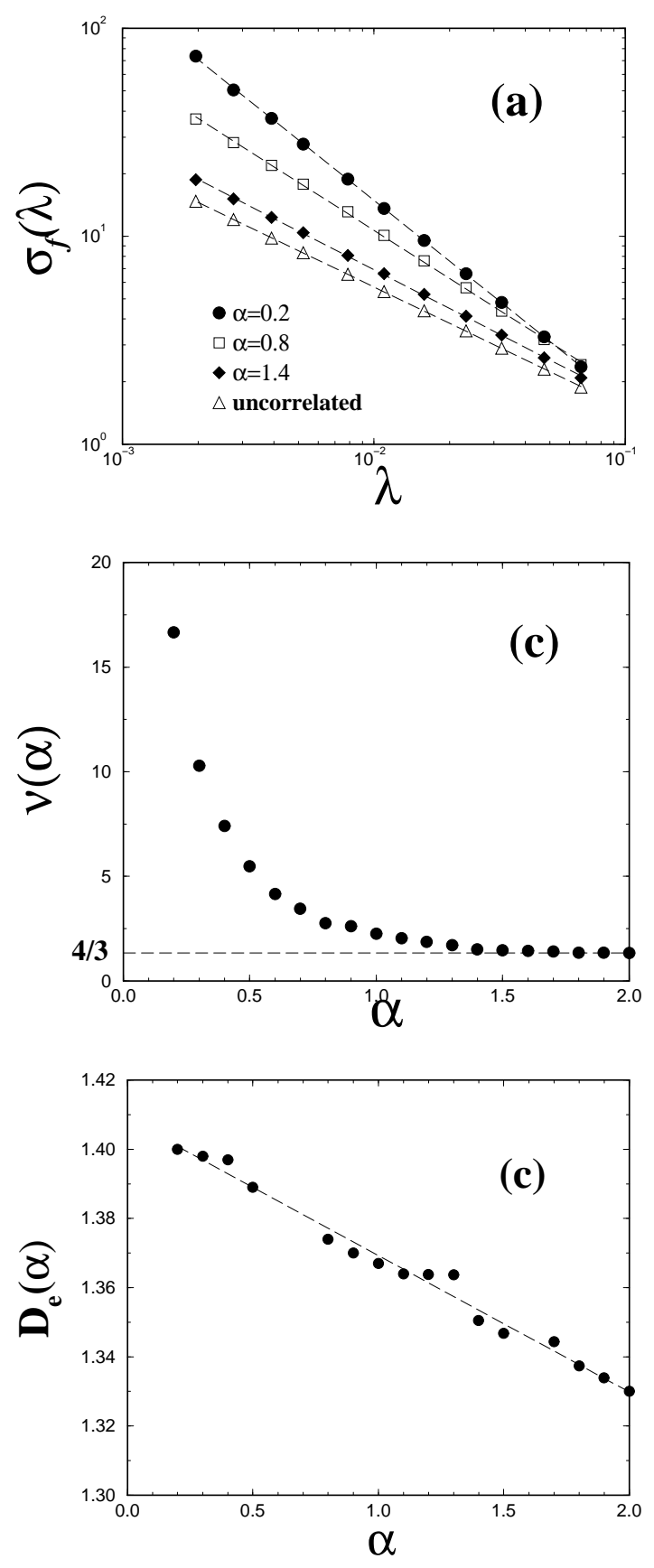

FIG. 3. (a) Width of the external perimeter as a function of the density gradient, $\sigma_{f}(\lambda)$, for several degree of correlations. (b) Connectedness length exponent $\nu(\alpha)$ as a function of the correlation exponent $\alpha$ calculated from Fig. 3a using Eq. (11). The value $\nu=4 / 3$ corresponds to the uncorrelated percolation problem $(\alpha=2)$. (c) Fractal dimension of the external perimeter of the largest cluster as a function of the degree of correlation, $D_{e}(\alpha)$.

We have analyzed the distribution of areas of actual cities, such as the system of cities surrounding London and Berlin for different years (Fig. 2a), and also we analyzed the area distribution of urban systems at larger scales by using the data of all settlements of Great Britain for the years 1981 and 1991 [28]. In the case of the towns around Berlin and London, we first digitize the empirical data of Fig. 4.1 of Ref. [27] (Berlin 1920 and 1945, shown in the last two panels of Fig. 2a), and Fig. 10.8 of Ref. [6] corresponding to London 1981. Then, we count the number of towns that are covered by $A$ sites, putting the result in logarithmically spaced bins (of size $1.2^{k}$, with $k=1,2, \ldots)$, and averaging over the size of the bin.

We calculate the actual distribution of the areas of the urban settlements around Berlin and London, and find (Fig. 5 a) that for both cities, $N(A)$ follows a power-law with exponent close to 2

$$
\begin{gathered}
N(A) \sim A^{-1.98} \quad \text { [Berlin, 1920, 1945] } \\
N(A) \sim A^{-1.96} \quad \text { [London, 1981]. }
\end{gathered}
$$

Figure $5 \mathrm{~b}$ shows the distribution of all urban areas in Great Britain for the years 1981 and 1991.. We find a power-law with an exponent consistent with the data of London and Berlin at smaller scales:

$$
N(A) \sim A^{-2.03} \quad \text { [Great Britain, 1981, 1991]. }
$$

Other studies have recently confirmed the validity of these results for larger length scales and also for the population distributions which is known to scale as the occupied area 29].

These results can be understood in the context of our model. Insight into this distribution can be developed by first noting that the small clusters surrounding the largest cluster are all situated at distances $r$ from the $\mathrm{CBD}$ such that $p(r)<p_{c}$ or $r>r_{f}$. Therefore, we find $N(A)$, the cumulative area distribution of clusters of area $A$, to be

$$
N(A)=\int_{0}^{p_{c}} n(A, p) d p \sim A^{-\left(\tau+1 / d_{f} \nu\right)} .
$$

Here,

$$
n(A, p) \sim A^{-\tau} g\left(A / A_{0}\right)
$$

is defined to be the average number of clusters containing $A$ sites for a given $p$ at a fixed distance $r$, and $\tau=1+2 / d_{f}$. Here,

$$
A_{0}(r) \sim \xi(r)^{d_{f}} \sim\left|p(r)-p_{c}\right|^{-d_{f} \nu}
$$

corresponds to the maximum typical area occupied by a cluster situated at a distance $r$ from the CBD, while $g\left(A / A_{0}\right)$ is a scaling function that decays rapidly (exponentially) for $A>A_{0}$.

In our numerical simulations we find a drastic increase of $\nu(\alpha)$ with the increase of the long-range correlations $(\alpha \rightarrow 0)$ (Fig. 3b) The connectedness exponent $\nu(\alpha)$ affects the area distribution of the small clusters around the 
CBD (Fig. (4), as specified by Eq. (18), and can be used to quantify the degree of interaction between the CBD and the small surrounding towns. For instance, for a strongly correlated system of cities characterized by small $\alpha, \nu(\alpha)$ is large so that the area $A_{0}(r)$ and the linear extension $\xi(r)$ of the towns will be large even for towns situated away from the CBD. This effect is observed in the correlated systems of cities of Fig. 1. 1 .

In Fig. 5 a we plot the power-law for the area distribution predicted by Eq. (18) along with the real data for Berlin and London and all Great Britain. In particular, the slope predicted for the uncorrelated system is

$$
N(A) \sim A^{-2.45}, \quad \text { [uncorrelated model] }
$$

while for the strongly correlated model is

$$
N(A) \sim A^{-2.06}, \quad[\text { strongly correlated model]. }
$$

Therefore, we find that the power law of the area distribution of actual cities are consistent with the prediction (dashed line, Fig. 5a) for the case of highly correlated systems. These results quantify the qualitative agreement between the morphology of actual urban areas and the strongly correlated urban systems obtained in our simulations. Clearly, the exponent of the area distribution provides a stronger test of our model against observations than does the fractal dimension $D_{e}$ of the perimeter.

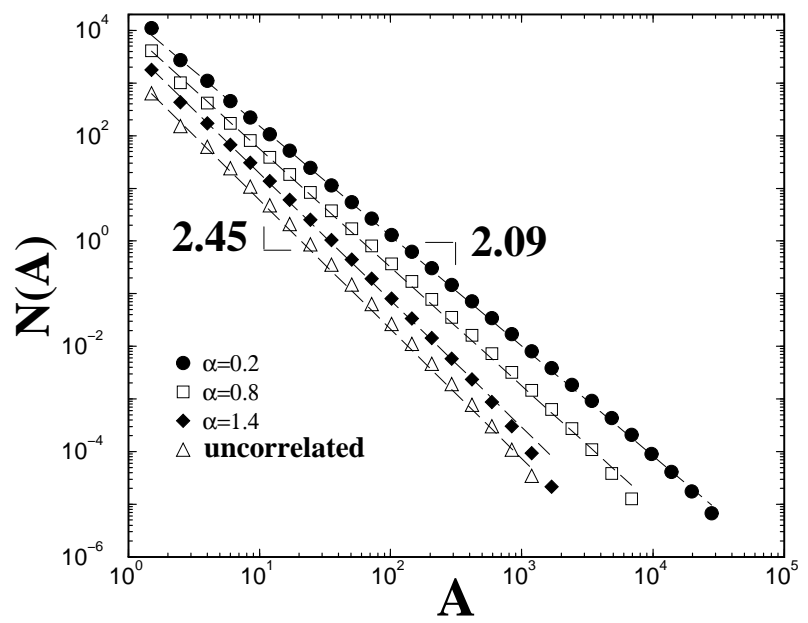

FIG. 4. Log-log plot of the area distribution function $N(A)$ calculated for the present model for different degrees of correlation. From top to bottom, $\alpha=0.2, \alpha=0.8, \alpha=1.4$, and uncorrelated case. The linear fits correspond to the predictions of Eq. (18) using the values of $\nu(\alpha)$ from Fig. 3 $\mathrm{b}$, and $d_{f}=1.89$.

\section{DYNAMICS}

We now discuss a generalization of our static model to describe the dynamics of urban growth. Empirical studies [15] of the population density profile of cities show a remarkable pattern of decentralization, which is quantified by the decrease of $\lambda(t)$ with time (see Table 4 in Ref. [30], and Fig. 6). Therefore the dynamics in the model are quantified by a decreasing $\lambda(t)$, as occurs in actual urban areas. In the context of our model, this flattening pattern can be explained as follows. The model of percolation in a gradient can be related to a dynamical model of units (analogous to the development units in actual cities) diffusing from a central seed or core [21 23]. In this dynamical system, the units are allowed to diffuse on a two-dimensional lattice by hopping to nearest-neighbor positions. The density of units at the core remains constant: whenever a unit diffuses away from the core, it is replaced by a new unit.
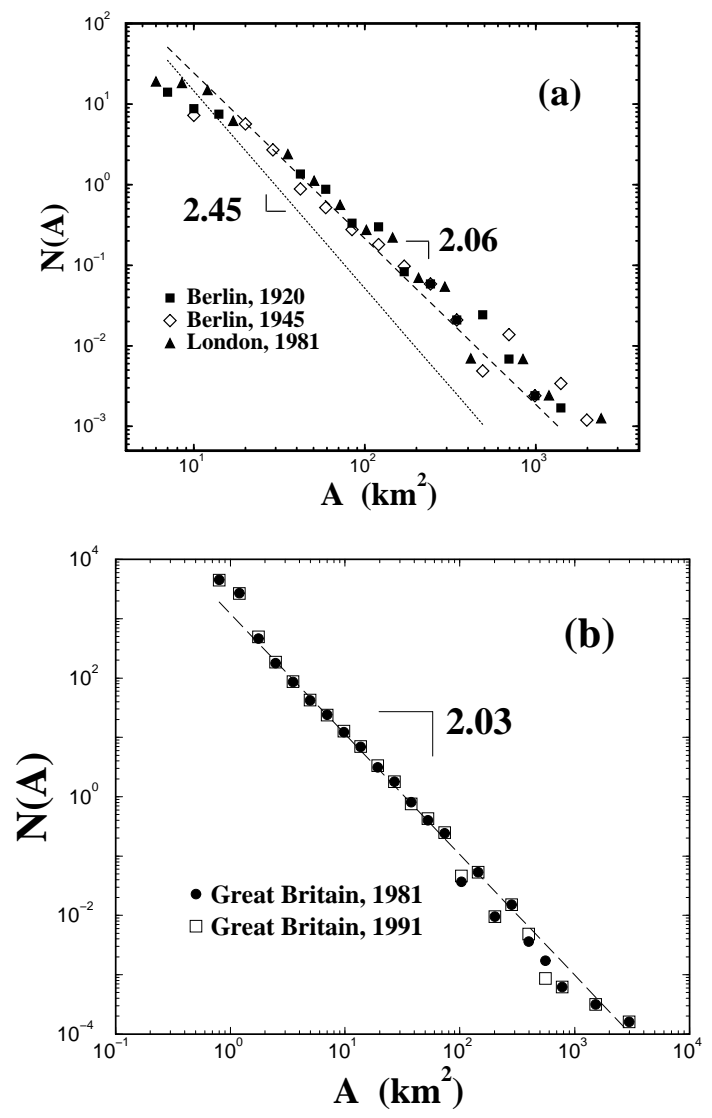
FIG. 5. (a) Log-log plot of the area distribution $N(A)$ of the actual towns around Berlin and London. We first digitize the empirical data of Fig. 4.1 of Ref. 27. (Berlin 1920 and 1945, shown in the last two panels of Fig. 2a), and Fig. 10.8 of Ref. [6] (London 1981). Then, we count the number of towns that are covered by $A$ sites, putting the result in logarithmically spaced bins (of size $1.2^{k}$, with $k=1,2, \ldots$ ), and averaging over the size of the bin. A power-law is observed for the area distributions of both urban systems. The dotted line shows the predictions of our model for the uncorrelated case $($ slope $=2.45)$, while the dashed line gives results for the strongly correlated case $($ slope $=2.06)$. Note that the area distributions for both cities agree much better with the strongly correlated case $(\alpha \rightarrow 0)$. (b) Log-log plot of the area distribution of all the urban areas in Great Britain in 1981 and 1991. The data fits to a power law of exponent 2.03. Notice also the very small changes of the urban areas in a 10 year period.

The density of units can be mapped to the density of occupied urban areas

$$
\rho_{A}(r)=e^{-\lambda r}
$$

which in turn is proportional to the population density $\rho(r)$ [6]. A well-defined diffusion front, defined as the boundary of the cluster of units that is linked to the central core, evolves in time. The diffusion front corresponds to the urban boundary of the central city. The static properties of the diffusion front of this system were found to be the same as those predicted by the gradient percolation model 21 23. Moreover, the dynamical model can explain the decrease of $\lambda(t)$ with time observed empirically. As the diffusion front situated around $r_{f}$ moves away from the core, the city grows and the density gradient decreases since $\lambda(t) \propto 1 / r_{f}$.

These considerations are tested in Fig. 2b, which shows our dynamical urban simulations of a strongly interacting system of cities characterized by a correlation exponent $\alpha=0.05$ for three different values of $\lambda$ obtained from the data of Berlin from Fig. 6. Qualitative agreement is observed between the morphology of the cities and towns of the actual data of Fig. $2 a$ and the simulations of Fig. $2 b$.

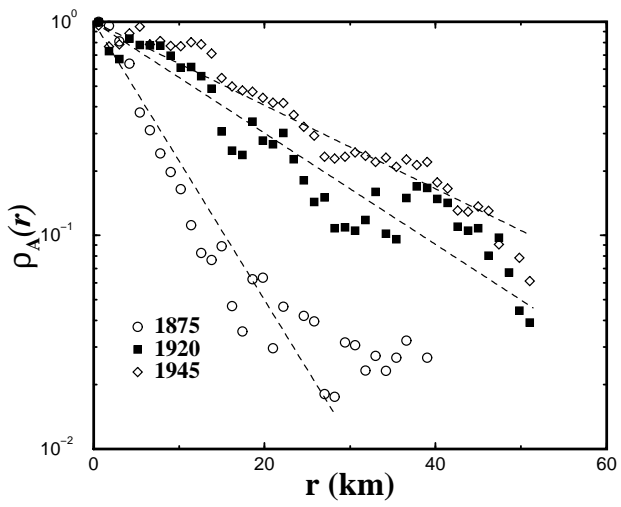

FIG. 6. Semi-log plot of the density of occupied urban areas $\rho_{A}(r)=e^{-\lambda r}$ for the three different stages in the growth of Berlin shown in Fig. A $a$. Least square fits yield the results $\lambda \simeq 0.030, \lambda \simeq 0.012$, and $\lambda \simeq 0.009$, respectively, showing the decrease of $\lambda$ with time. We use these density profiles in the dynamical simulations of Fig. $6 b$.

\section{DISCUSSION: URBAN PLANNING}

Throughout this century, the dominant planning policy in many western nations has been the containment of urban growth. This has been effected using several instruments, particularly through the siting of new settlements or new towns at locations around the growing city which are considered to be beyond commuting distance, but also through the imposition of local controls on urban growth, often coordinated regionally as "green belts" 31. One of the key elements in the growth models we have proposed here is the characteristic length scale over which growth takes place. In the case of the gradient percolation model, correlations occur over all length scales, and the resulting distributions are fractal, at least up to the percolation threshold.

In examining the changing development of Berlin in Fig. 2a, it appears that the fractal distribution remains quite stable over a period of 70 years and this implies that any controls on growth that there might have been do not show up in terms of the changing settlement pattern, implying that the growth dynamics of the city are not influenced by such control. A rather different test of such policies is provided in the case of London where a green belt policy was first established in the 1930s and rigorously enforced since the 1950s. The question is whether this has been effective in changing the form of the settlement pattern. First, it is not clear that the siting of new towns beyond London's commuting field was ever beyond the percolation field and thus it is entirely possible that the planned new settlements in the 1950s and 1960s based on existing village and town cores simply reinforced the existing fractal pattern.

In the same manner, the imposition of local controls on growth in terms of preserving green field land from development seems to have been based on reinforcing the kind of spatial disorder consistent with morphologies generated through correlated percolation. The regional green belt policy was based on policies being defined locally and then aggregated into the green belt itself, and this seems to suggest that the morphology of nondevelopment that resulted was fractal. This is borne out in a fractal analysis of development in the London region which suggests that the policy has little impact on the overall morphology of the area [6, 32]. Moreover, we note that the coincidence between the settlement area distribution for different cities and different years (Berlin 1920 and 1945, and London 1981) suggests that local planning policies such as the green belt may have a relatively low impact on the distribution of towns. Our model suggests 
that the area distribution is determined by the degree of interactions among development units, and that its scaling properties are independent of time. Current debates on urban growth have now shifted to the development of brownfield sites in cities, and it would be interesting to quantify the extent to which such future developments might reinforce or counter the "natural" growth of the city as implied in these kinds of models.

To develop more detailed and conclusive insights into the impact of urban policies on growth, it is necessary to develop the model further. This model implies that the area and size distributions, the degree of interaction amongst dependent units of development, and fractal dimension are independent of time. The only time dependent parameter is the gradient $\lambda$ and it appears that we might predict future urban forms simply by extrapolating the value of $\lambda$ in time. However, we have yet to investigate the influence of topography and other physical constraints on development, the influence of transport routes and the presence of several "independent" central cores or CBDs in the urban region.

These models can also be further adapted to predict bond as well as site percolation and in future work we will explore the extent to which such interactions between sites and cities might be modeled explicitly. Our interest in such examples is in the universality of the exponents that we have demonstrated here, and which we wish to relate to the impact of urban planning policies.

ACKNOWLEDGEMENTS: We thank NSF and CNPq for financial support.

[1] W. Christaller, Central Places in Southern Germany (Prentice-Hall, Englewood Cliffs, NJ, 1933, trans. 1966).

[2] G. K. Zipf, Human Behavior and the Principle of Least Effort (Harvard University Press, Cambridge, MA, 1949).

[3] J. Q. Stewart and W. Warntz, J. Region. Sci. 1, 99 (1958).

[4] M. J. Beckmann, Location Theory (Random House, New York, 1968).

[5] P. R. Krugman, The Self-Organizing Economy (Blackwell Publishers, Cambridge, 1996).

[6] M. Batty and P. Longley, Fractal Cities (Academic Press, San Diego, 1994).
[7] D. Stauffer and A. Aharony, Introduction to Percolation Theory (Taylor and Francis, Philadelphia, 1994).

[8] J.-F. Gouyet, Physics and Fractal Structures (SpringerVerlag, Berlin, 1995).

[9] A. Bunde and S. Havlin, eds., Fractals and Disordered Systems, 2nd ed. (Springer-Verlag, Berlin 1996).

[10] H. A. Makse, S. Havlin, and H. E. Stanley, Nature 377, 608 (1996).

[11] L. Benguigui, M. Daoud, Geog. Analy. 23, 362 (1991).

[12] L. Benguigui, Physica A 219, 13 (1995).

[13] T. A. Witten and L. M. Sander, Phys. Rev. Lett. 47, 1400 (1981).

[14] T. Vicsek, Fractal Growth Phenomena, 2nd ed. (World Scientific, Singapore 1991).

[15] C. Clark, J. R. Stat. Soc. (Series A) 114, 490 (1951).

[16] A. Coniglio, C. Nappi, L. Russo, and F. Peruggi, J. Phys. A 10, 205 (1977).

[17] A. Weinrib, Phys. Rev. B 29, 387 (1984); A. Weinrib and B. I. Halperin, Phys. Rev. B 27, 413 (1983).

[18] S. Prakash, S. Havlin, M. Schwartz, and H. E. Stanley, Phys. Rev. A 46, R1724 (1992).

[19] H. A. Makse, S. Havlin, H. E. Stanley, and M. Schwartz, Chaos, Solitons, and Fractals 6, 295 (1995); H. A. Makse, S. Havlin, P. Ch. Ivanov, P. R. King, S. Prakash, and H. E. Stanley, Physica A 233, 587-605 (1996).

[20] H. A. Makse, S. Havlin, M. Schwartz, and H. E. Stanley, Phys. Rev. E 53, 5445 (1996).

[21] B. Sapoval, M. Rosso, and J.-F. Gouyet, J. Phys. Lett. 46, L149 (1985).

[22] M. Rosso, J.-F. Gouyet, and B. Sapoval, Phys. Rev. Lett. 57, 3195 (1986).

[23] M. Kolb, T. Gobron, J.-F. Gouyet, B. Sapoval, Europhys. Lett. 11, 601 (1990).

[24] D. Saupe in The Science of Fractal Images H.-O. Peitgen and D. Saupe, eds. (Springer-Verlag, New York 1988);

[25] J. Feder, Fractals (Plenum Press, New York 1988).

[26] C.-K. Peng et al. Phys. Rev. A 44, 2239 (1991).

[27] P. Frankhauser, La Fractalité des Structures Urbaines (Collection Villes, Anthropos, Paris 1994).

[28] Source: The 1991 Census, Crown Copyright. ESRC/JISC purchase. http://www.geog.leeds.ac.uk/staff/i.turton/msc/msc.htm

[29] D. Zanette and S. Manrubia, Phys. Rev. Lett. 79, 323 (1997).

[30] E. S. Mills and J. P. Tan, Urban Studies 17, 313 (1980).

[31] R. J. C. Munton, London's Green Belt: Containment in Practice (Allen and Unwin, London, 1983).

[32] J. Shepherd, P. A. Longley, M. Batty, and G. Sadler, Regional Studies 26, 437 (1992). 